\title{
Temporal dynamics of spatially localized waves in quadratic nonlinear waveguide arrays
}

\author{
Frank Setzpfandt, ${ }^{1,2, *}$ Alexander S. Solntsev, ${ }^{1}$ Dragomir N. Neshev, ${ }^{1}$ Thomas Pertsch, ${ }^{2}$ Wolfgang Sohler, ${ }^{3}$ and Roland Schiek ${ }^{4}$ \\ ${ }^{1}$ Nonlinear Physics Center, Research School of Physics and Engineering, Australian National University, Canberra, 0200 ACT, Australia \\ ${ }^{2}$ Institute of Applied Physics, Abbe Center of Photonics, Friedrich-Schiller-Universität Jena, Max-Wien-Platz 1, 07743 Jena, Germany \\ ${ }^{3}$ Applied Physics, University of Paderborn, Warburger Strasse 100, 33095 Paderborn, Germany \\ ${ }^{4}$ University of Applied Sciences Regensburg, Prüfeninger Strasse 58, 93049 Regensburg, Germany
}

(Received 18 January 2014; published 31 March 2014)

\begin{abstract}
We study experimentally and theoretically the temporal dynamics of laser pulses propagating under conditions of spatial self-focusing in quadratic nonlinear waveguide arrays made from periodically poled lithium niobate. We observed temporal pulse breakup and temporal pulse narrowing and studied the dynamics of these effects in different waveguides. We investigated the influence of the frequency dependence of the mode indices as a limiting factor for soliton formation. Our experimental results are in good agreement with the theoretical model developed from coupled-mode theory, providing a detailed understanding of pulse dynamics and beam distribution in waveguide arrays with quadratic nonlinearity.
\end{abstract}

DOI: 10.1103/PhysRevA.89.033863

PACS number(s): 42.65.Tg, 42.65.Sf, 42.65.Ky

\section{INTRODUCTION}

Nonlinear waveguide arrays (WGA) are considered to be important devices for all-optical circuits $[1,2]$. To date, many experiments in nonlinear WGAs, including the demonstrations of discrete solitons $[3,4]$, have been conducted by using continuous-wave $(\mathrm{cw})$ beams. The use of pulsed-laser sources with high peak powers strongly benefits nonlinear processes, but even experiments with pulsed light were often interpreted within the quasi-cw approximation [5-7]. Measurements of the complete nonlinear spatiotemporal dynamics of short pulses, revealing the effects of the frequency dependence of the nonlinearity, have been hindered by difficulties in the experimental characterization of the temporal output profiles of short pulses. However, the practical implementation of nonlinear waveguide devices for applications in all-optical switching and ultrafast optical signal processing requires a detailed characterization of the full spatial and temporal dynamics of optical pulses in these device.

While some important experiments on spatiotemporal nonlinear effects of short pulses have already been performed, most of the work has concentrated on WGAs with cubic nonlinearity. Examples include nonlinear pulse reshaping [8,9], discrete spatiotemporal $X$ waves [10,11], and discrete light bullets [12-15]. However, in all cubic nonlinear experiments the required peak powers for operation remain high.

On the other hand, WGAs with quadratic nonlinear response allow for nonlinear effects at orders-of-magnitude lower powers [7]. This is achieved through the so-called cascading nonlinearity [16], where the generation of a second harmonic $(\mathrm{SH})$ field and its back conversion to the fundamental wave $(\mathrm{FW})$ of twice the $\mathrm{SH}$ wavelength result in an overall nonlinear phase shift similar to the one generated by a cubic nonlinearity. Recently, the measurement of pulse spectra in quadratic nonlinear WGAs has led to theoretical predictions of unusual temporal pulse dynamics [17], which are awaiting experimental verification. While temporal reshaping of pulsed all-optical switching was already characterized in two coupled

\footnotetext{
*f.setzpfandt@uni-jena.de
}

waveguides [18], the characterization of the full spatiotemporal dynamics of optical signals in quadratic WGAs has not yet been reported. Important questions on the limiting effects of waveguide dispersion, group-velocity mismatch (GVM), and bandwidth of the nonlinear response remain to be experimentally characterized. In this work, for the first time to our knowledge, we conduct direct pulse measurements in WGAs with quadratic nonlinearity and analyze the spatiotemporal reshaping of picosecond-long pulses. We reveal experimentally that the strongest effect on pulse reshaping and a limitation of discrete soliton formation comes from the frequency dependence of the effective indices of the interacting modes yielding different phase-matching conditions within the nonlinearly broadened pulse spectra. Our experimental results are well explained by a theoretical model.

The remainder of this paper is organized as follows: Our samples and experimental apparatus are introduced in Sec. II. We then discuss the numerical experiments, revealing the pulse reshaping mechanism in Sec. III and presenting the experimental confirmation of these findings in Sec. IV. Finally, we summarize the results in Sec. V.

\section{EXPERIMENTAL ARRANGEMENTS}

The system under investigation is described in Fig. 1. WGA samples as shown schematically in Fig. 1(a) are fabricated by indiffusion of 7- $\mu \mathrm{m}$-wide and 95-nm-thick titanium stripes at $1060{ }^{\circ} \mathrm{C}$ for 8.5 hours in an $L=71$-mm-long $Z$-cut congruent lithium niobate $\left(\mathrm{LiNbO}_{3}\right)$ crystal [19]. The propagation direction is along the crystallographic $\mathrm{X}$ axis. Highly uniform arrays are formed by 101 waveguides with a constant waveguide separation (center-to-center distance between the waveguides) of $d=15 \mu \mathrm{m}$. For the TM polarization used, the waveguides guide only one mode at the FW near $1550 \mathrm{~nm}$ and are multimoded in the SH-wavelength regime [20,21]. Numerically calculated intensity profiles of the $\mathrm{TM}_{00}$ modes at both wavelengths, which correspond very well to measured profiles, are shown in Fig. 1(b). The FW modes from neighboring waveguides are coupled due to their evanescent-field overlap. In contrast, the $\mathrm{SH} \mathrm{TM} 00$ mode at a wavelength near $775 \mathrm{~nm}$ shows a much stronger localization than the FW mode 
(a)

(b)

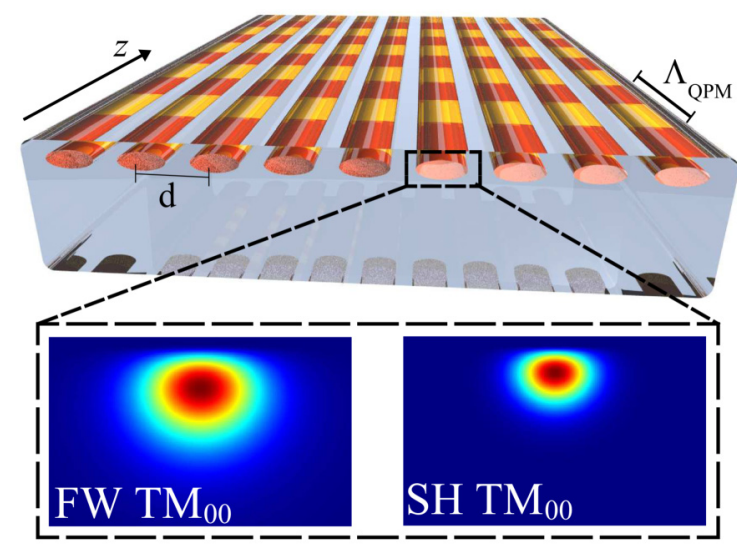

(c)

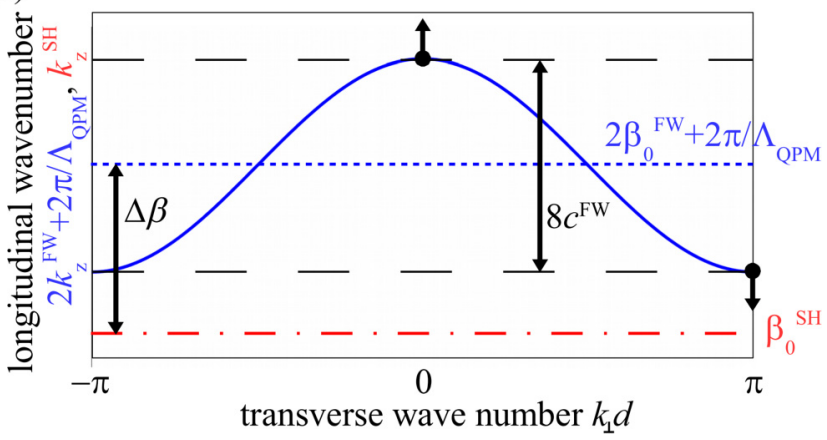

FIG. 1. (Color online) (a) Sketch of waveguide array with period $d$. The red and yellow shading denotes the different crystal orientations, which form the quasi-phase-matching grating with a period $\Lambda_{\mathrm{QPM}}$. (b) FW and $\mathrm{SH} \mathrm{TM}_{00}$ modes in an isolated array waveguide. (c) The diffraction relation of FW (solid blue line) and $\mathrm{SH}$ (red dash-dotted line) array supermodes. $\Delta \beta$ indicates the phase mismatch between the SH band and an isolated array waveguide mode (dotted blue line) at the FW. The black arrows emerging from the FW band denote the bifurcation points of unstaggered (upwards) solitons for focusing nonlinearity and staggered (downwards) solitons for the defocusing nonlinearity.

[see Fig. 1(b)] and the linear coupling between neighboring SH $\mathrm{TM}_{00}$ modes can be neglected [7,20]. Therefore, the WGA dispersion relation $k_{z}\left(k_{\perp}\right)$ describing the dependence of the longitudinal wave vector $k_{z}$ on the transversal wave vector $k_{\perp}$ of the array supermodes is very different for FW and SH. In Fig. 1(c), we schematically plot FW and SH dispersion relations with the solid blue and dash-dotted red lines, respectively. While the longitudinal wave vector of the $\mathrm{SH}$ array supermode $k_{z}^{\mathrm{SH}}$ is constant and equal to the propagation constant of a single-waveguide mode $\beta_{0}^{\mathrm{SH}}$, the wave numbers of the FW supermodes form a band, as is well known in the physics of periodic photonic structures [22,23]. In the case of weak diffraction this band is approximated by the often-used cosine form $[20,23]$

$$
k_{z}^{\mathrm{FW}}=\beta_{0}^{\mathrm{FW}}+2 c^{\mathrm{FW}} \cos \left(k_{\perp} d\right),
$$

with the propagation constant $\beta_{0}^{\mathrm{FW}}$ of the $\mathrm{FW} \mathrm{TM}_{00}$ mode in a single waveguide and a coupling constant $c^{\mathrm{FW}}=\pi /\left(2 L_{\mathrm{c}}^{\mathrm{FW}}\right)$. For the WGA with $d=15 \mu \mathrm{m}$ that was used in our experiments, the coupling length at a wavelength of $1557.3 \mathrm{~nm}$ is measured to be $L_{\mathrm{c}}^{\mathrm{FW}}=15.8 \mathrm{~mm}$, which corresponds to a coupling strength of $c^{\mathrm{FW}}=99.4 / \mathrm{m}$. This coupling strength is considered to be constant across the wavelength range used in our experiments.

The nonlinear interaction between the FW and SH fields is determined by the phase mismatch $\Delta \beta$, which is defined as

$$
\Delta \beta=2 \beta_{0}^{\mathrm{FW}}-\beta_{0}^{\mathrm{SH}}+\frac{2 \pi}{\Lambda_{\mathrm{QPM}}} .
$$

$\Delta \beta$ is also indicated in Fig. 1(c). Here, $\Lambda_{\mathrm{QPM}}$ is the period of the electric-field-poled quasi-phase-matching (QPM) grating of the sample $[24,25]$, which periodically switches the orientation of the crystal axis along the propagation direction. The additional wave vector introduced by the periodic sign change of the nonlinear coefficient $\chi^{(2)}$ can compensate the wave vector mismatch introduced by material and waveguide dispersion. The period of the QPM grating at room temperature is $\Lambda_{\mathrm{QPM}}=16.751 \mu \mathrm{m}$. However, to minmize photorefraction we heat the sample to $230{ }^{\circ} \mathrm{C}$. Due to thermal expansion $\Lambda_{\mathrm{QPM}}$ increases to $16.805 \mu \mathrm{m}$, leading to phase matching of second-harmonic generation (SHG) between the $\mathrm{FW}_{00}$ and the $\mathrm{SH}_{00}$ modes at an $\mathrm{FW}$ wavelength of $1557 \mathrm{~nm}$. Due to the temperature and wavelength dependence of the mode-propagation constants, the normalized phase mismatch $\Delta \beta L$ can be adjusted in our QPM- $\mathrm{LiNbO}_{3}$ waveguides by varying the $\mathrm{FW}$ wavelength or the crystal's temperature.

In the nonlinear experiments, the $\mathrm{FW}$ is converted to $\mathrm{SH}$. However, for nonzero phase mismatch the $\mathrm{SH}$ is back converted to the FW after the phase-coherence length $l_{\mathrm{pc}}=$ $\pi /|\Delta \beta|$ due to the different phase velocities of FW and SH. The subsequent generation and back mixing of the SH leads to a phase shift of the FW [26-29]. The physical consequences of the phase shift induced by the back-converted SH are similar to those of the nonlinear refractive phase shift induced by the Kerr nonlinearity. The "cascaded" process of simultaneous SHG and back conversion is called cascaded second-order nonlinearity [30] and mimics an effective Kerr nonlinearity. The cascaded nonlinearity is more flexible than a Kerr nonlinearity because its sign and strength can be adjusted by changing the phase mismatch. For a positive phase mismatch the effective cascading nonlinearity acting on the FW is positive and focusing, whereas it is negative or defocusing for a negative phase mismatch. Discrete spatial solitons can be excited in both scenarios $[7,31,32]$. In the first case they bifurcate from the top of the bands with a transverse wave number of $k_{\perp}=0$. In the second case they are generated from the bottom of the band at $k_{\perp}= \pm \pi / d$. Both situations are indicated by the black arrows in Fig. 1(c). However, due to the dependence of the cascaded nonlinearity on the wavelength-dependent phase mismatch, the nonlinearity becomes strongly frequency dependent and no longer acts instantaneous. The consequences for ultrashort pulse propagation are carefully discussed in Refs. [33,34] and related works. The aim of this contribution is to experimentally explore the spatiotemporal dynamics of discrete spatial solitons in the case of pulsed excitation, when the noninstantaneous character of the nonlinearity starts to influence the dynamics. Experiments were carried out with pulse lengths of a few ps. The corresponding spectral width allows us to observe spatial soliton formation close to the phase-matching wavelength but already enables the investigation of effects induced by varying phase mismatch across the spectrum. 

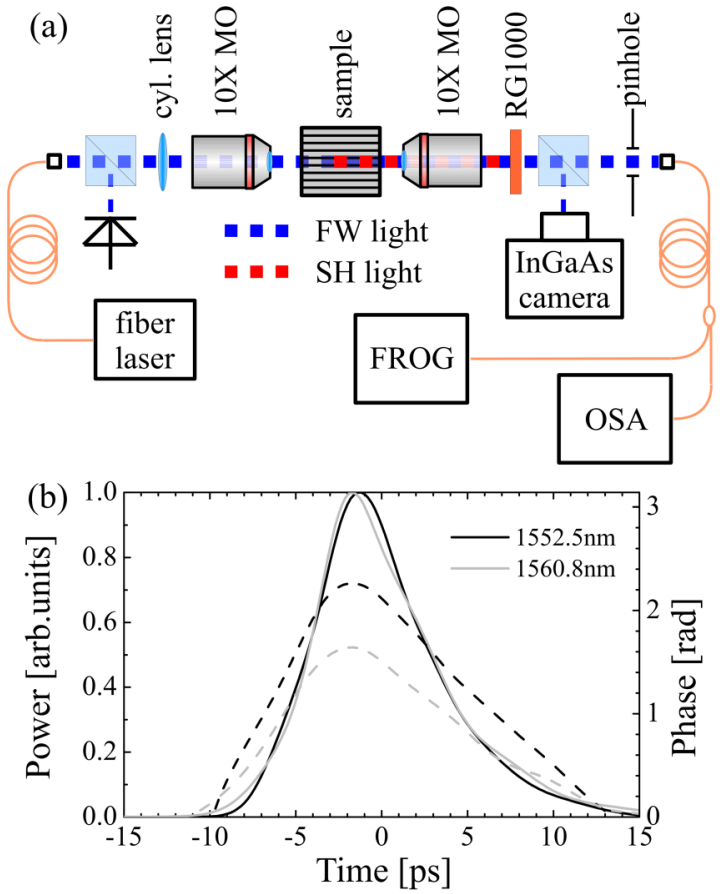

FIG. 2. (Color online) (a) Scheme of experimental setup showing the laser and beam shaping, the coupling to and from the sample with microscope objectives (MO), and the pulse-analysis setup. (b) Intensity (solid lines) and phase (dashed lines) of the input pulses at $\lambda=1552.5 \mathrm{~nm}$ and $\lambda=1560.8 \mathrm{~nm}$.

The spatiotemporal intensity distribution of the FW part of the signal transmitted through the WGA is characterized using the experimental setup shown in Fig. 2(a). Optical pulses with a pulse length of $7.4 \mathrm{ps}$ full width at half maximum (FWHM) were generated with an erbium-doped fiber laser and amplifier system from Pritel at a repetition rate of $5 \mathrm{MHz}$. The waveguide input is monitored by splitting off a small fraction of the input beam and measuring the power with an indium-gallium-arsenide (InGaAs) photodiode. To excite several waveguides of the WGA simultaneously, the input beam is shaped with a $300-\mathrm{mm}$-focal-length cylindrical lens. Together with a $10 \times$ coupling objective this forms a telescope for only the horizontal beam axis, leading to an elliptic input beam spot with a horizontal (vertical) FWHM of $42 \mu \mathrm{m}$ $(4 \mu \mathrm{m})$. The output of the WGA is filtered with an RG1000 filter to suppress the generated $\mathrm{SH}$. The $\mathrm{FW}$ is partly routed to an InGaAs camera which captures the time-integrated spatial intensity distribution of the output of the WGA. To measure the absolute output power, the camera can be replaced by an InGaAs photodiode. The FW output is spatially filtered by a pinhole which is placed in the image plane of the coupling objective. By this aperture one waveguide is selected for further characterization. The light from the selected waveguide is coupled to a single-mode fiber and routed either to an optical spectrum analyzer (OSA) or a frequency-resolved optical gating (FROG) pulse-measurement device based on SHG [35]. The temporal profile of the pulse is numerically retrieved from the FROG trace [36]. For double checking the measured FROG traces, their frequency marginals were compared with independently measured spectra [37]. The amplitude and the phase distribution of the input pulses were also measured with the FROG and used as input conditions in the simulations. These are shown in Fig. 2(b) for the wavelengths used in the experiments reported here. The pulse shape shows a single intensity maximum with a slightly asymmetric tail and does not show a dependence on wavelength. The small positive chirp of the pulse changes with wavelength. The time direction and the sign of the pulse's phase, which are not uniquely defined by SHG-FROG measurements, are determined by simulations showing either spectral broadening or narrowing as explained below in Sec. IV.

\section{NUMERICAL SIMULATION}

For a complete understanding of the experimental results and to obtain information about the pulse dynamics inside the sample, we conduct numerical simulations of the pulse propagation. Nonlinear propagation in the WGA is described by the coupled mode equations

$$
\begin{gathered}
\left(i \frac{\partial}{\partial z}-\frac{D^{\mathrm{FW}}}{2} \frac{\partial^{2}}{\partial t^{2}}+\beta^{\mathrm{FW}}(z)+i \frac{\alpha^{\mathrm{FW}}}{2}\right) u_{n}^{\mathrm{FW}} \\
+c^{\mathrm{FW}}\left[u_{n+1}^{\mathrm{FW}}+u_{n-1}^{\mathrm{FW}}\right]=-\omega^{\mathrm{FW}} \chi_{\mathrm{eff}} u_{n}^{\mathrm{FW} *} u_{n}^{\mathrm{SH}}, \\
\left(i \frac{\partial}{\partial z}+i \delta \frac{\partial}{\partial t}-\frac{D^{\mathrm{SH}}}{2} \frac{\partial^{2}}{\partial t^{2}}+\beta^{\mathrm{SH}}(z)+i \frac{\alpha^{\mathrm{SH}}}{2}\right) u_{n}^{\mathrm{SH}} \\
=-\omega^{\mathrm{FW}} \chi_{\mathrm{eff}}\left[u_{n}^{\mathrm{FW}}\right]^{2} .
\end{gathered}
$$

Here the $u_{n}^{m}$ are the complex amplitudes of FW and SH in the $n$th waveguide, with the index $m$ denoting either FW or $\mathrm{SH}$. The dispersive characteristics of the WGA are taken into account through the group-velocity dispersion (GVD) coefficients $D^{\mathrm{FW}}=0.0015 \mathrm{ps}^{2} / \mathrm{cm}$ and $D^{\mathrm{SH}}=0.0044 \mathrm{ps}^{2} / \mathrm{cm}$. The GVM coefficient is defined as $\delta=1 / v_{g}^{\mathrm{SH}}-1 / v_{g}^{\mathrm{FW}}=$ $3.32 \mathrm{ps} / \mathrm{cm}$ with the group velocities $v_{g}^{m} \cdot \alpha^{\mathrm{FW}}=0.047 / \mathrm{cm}$ and $\alpha^{\mathrm{SH}}=0.092 / \mathrm{cm}$ are the linear loss coefficients. The $z$ dependence of $\beta^{m}(z)$ describes the linear inhomogeneity of the waveguides along the propagation direction due to temperature variations in the crystal oven and crystal inhomogeneities. Finally, the nonlinear interaction is accounted for by the effective nonlinearity $\chi_{\text {eff }}$ containing the mode overlap and the applicable second-order nonlinear susceptibility element $\chi_{z z z}^{(2)}=20.6 \mathrm{pm} / \mathrm{V}$ [38]. The coefficients describing the dispersion in the WGA are determined by wavelength-dependent numerical calculations of the mode-propagation constants based on a precise description of the WGA index profile $[39,40]$ and measured values for the refractive index of lithium niobate [41]. The system of Eqs. (3) and (4) is solved with the well-known split-step algorithm [42].

To identify the effect of dispersion on the nonlinearity we first compare numerical simulations of pulse propagation in an idealized system without GVM and GVD, and in the real WGA taking all experimental conditions into account. Both cases are compared in Fig. 3 (upper and bottom rows, respectively) for a positive phase mismatch resulting in an effective focusing nonlinearity. We consider a Gaussian spatial input distribution with a FWHM of 2.8 periods $d$, corresponding to the experimental beam width. The phase mismatch $\Delta \beta L=51 \pi$, which is also used in the experiments, is large enough such 

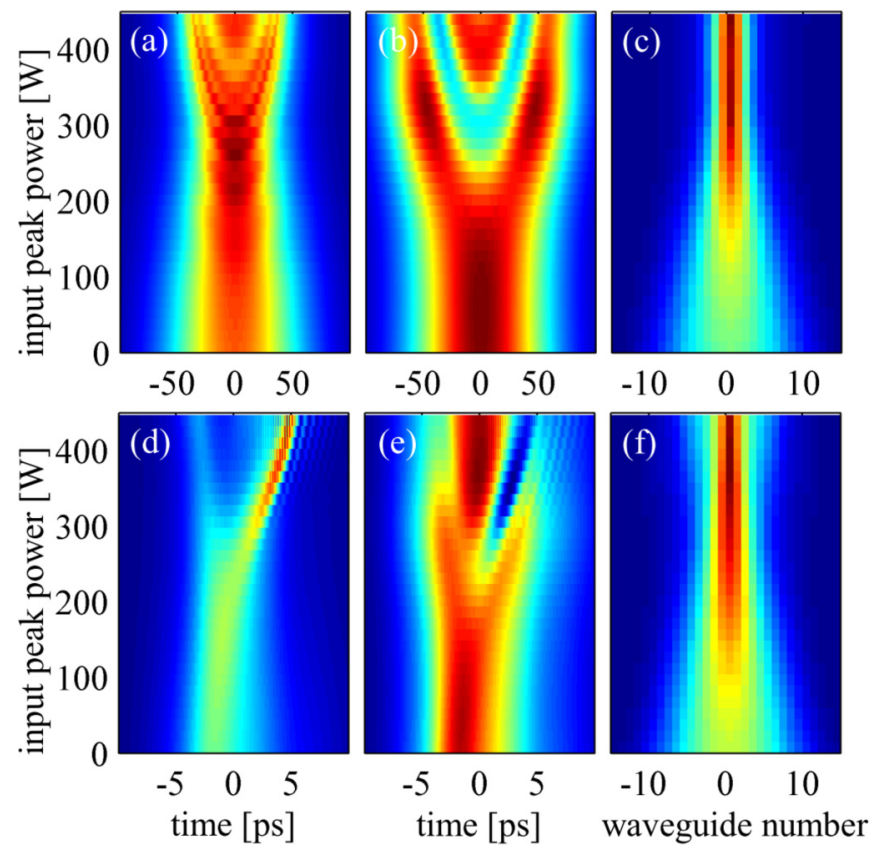

FIG. 3. (Color online) (a)-(c) Pulse propagation in a homogeneous WGA without GVM and GVD, with Gaussian pulse excitation of 100 ps FWHM pulse length, and a phase mismatch $\Delta \beta L=51 \pi$. Normalized power in (a) the central waveguide and (b) in the fourth waveguide from the center at the output of the WGA. (c) Normalized time-integrated power in all waveguides at the output of the WGA. (d), (e) Same as (a)-(c) in the WGA of our experiment with the experimental input pulse taking into account all experimental details, e.g., nonuniformities and dispersion.

that FW depletion is small and cascading is the dominant mechanism. On the other hand it is small enough to observe the sought-after effects for the experimentally available powers. In Fig. 3 we plot the normalized output powers vs the input peak power in the central waveguide [Figs. 3(a) and 3(d)] and, to represent pulse evolution in the spatial wings of the power distribution, in the fourth waveguide [Figs. 3(b) and 3(e)]. The evolution in other waveguides outside the central waveguide is qualitatively similar to the depicted one. Furthermore, we show the time-integrated output powers of all waveguides in Figs. 3(c) and 3(f). In Fig. 3, the plotted quantities for each input peak power are normalized to the corresponding pulse energy in the central [Figs. 3(a) and 3(d)], fourth [Figs. 3(b) and 3(e)], and all [Figs. 3(c) and 3(f)] waveguides, respectively. In the idealized system [Figs. 3(a)-3(c)] a Gaussian pulse with a length of 100 ps ensures that GVD can definitely be neglected. Setting the GVM to zero ensures a constant nonlinearity and especially prevents phase matching within the pulse spectrum. The resulting cascaded nonlinearity acts quasiinstantaneous. The waveguides are assumed to be uniform along the whole sample. The FW depletion is small with less than a total of $4 \%$ generated $\mathrm{SH}$ at the maximum power. A perfect symmetric picture indicates that the pulses in the array behave exactly according to their momentary power, like in the $\mathrm{cw}$ case. At the soliton power of 250 to $300 \mathrm{~W}$ the powerful pulse center is concentrated in the central waveguides, forming a spatial soliton, as seen in Figs. 3(a) and 3(c), respectively. The pulse here is shortened because, due to discrete diffraction, the lower-intensity pulse wings spread into the waveguides away from the center. A resulting pulse breakup in these waveguides is evident with the missing pulse center and the two remains of the pulse wings, as seen in Fig. 3(b). This situation is similar to the X waves reported in Ref. [10]; however, in our idealized simulation dispersion is neglected and the evolution is determined by discrete diffraction and nonlinearity only. Note that each pulse in the figures is normalized to its energy, and that the remains of the pulse wings in the outer waveguide have a much lower peak power than the narrow pulse in the center. When the input peak power exceeds the soliton power we observe a back switching of energy in Fig. 3(b), which has also been observed in two-core couplers [18]. However, in the output powers of the WGA in Fig. 3(c) the beam stays focused also for powers above the soliton power, indicating the formation of spatial solitons.

The simulation of the pulse propagation in the WGA sample with its realistic and nonideal properties uses the measured 7.4-ps-long pulses as input. Figures 3(d)-3(f) present the calculated dependencies where a different behavior is observed. The simulation was conducted for the same relatively large phase mismatch of $\Delta \beta L=51 \pi$ as above, and not more than $7 \%$ of the input energy is converted to $\mathrm{SH}$ at the highest input power. The most noticeable feature in Figs. 3(d) and 3(e) is the lack of temporal symmetry. The pulse in the center waveguide [Fig. 3(d)] becomes increasingly asymmetric and develops a sharply peaked and very powerful tail. This is due to cascaded SHG, which is generated more efficiently in the small wavelength wings of the broadened pulse spectrum. Because of the wavelength dependence (dispersion) of the effective mode indices, there always exists a wavelength where phase matching for SHG or sum-frequency generation occurs. Even when the cascaded nonlinearity is operated far from that phase-matching wavelength, for decreasing pulse length or for spectral broadening due to self-phase modulation, some spectral components are always driven towards phase matching, where $\mathrm{SH}$ is generated more efficiently. In the time domain the phase-matched SHG corresponds to a growing $\mathrm{SH}$ pulse that propagates with its slower group velocity behind the FW pulse and withdraws energy from the FW pulse [43]. This results in asymmetric depletion at the beginning of the FW pulse and back conversion at the pulse end, yielding a strongly asymmetric FW pulse, as seen in Fig. 3(d).

The pulses in waveguide 4, plotted in Fig. 3(e), show the pulse breakup. Furthermore, a more pronounced back switching with respect to the idealized case discussed above is observed. As seen in Fig. 3(f) these perturbations disturb the discrete spatial soliton, since for powers above the soliton power the beam width increases again and the energy fraction in the central waveguide decreases. For such powers the cascaded nonlinearity definitely deviates from an instantaneous cubic nonlinearity due to GVM. Here the cascaded nonlinearity becomes strongly frequency dependent and disturbed by significant energy transfer from FW to SH.

\section{EXPERIMENTAL RESULTS}

\section{A. Focusing nonlinearity}

To experimentally verify the characteristics of a noninstantaneous nonlinearity on the temporal pulse dynamics we 
(a)

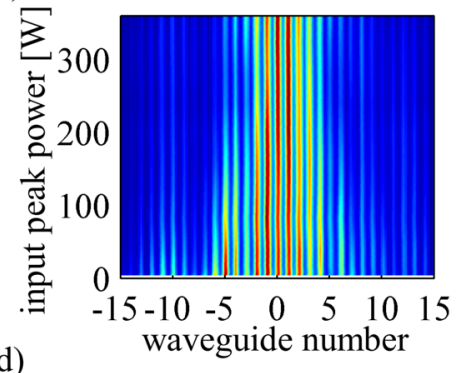

(b)

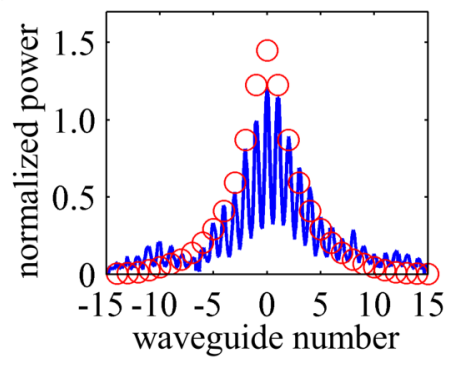

(c)

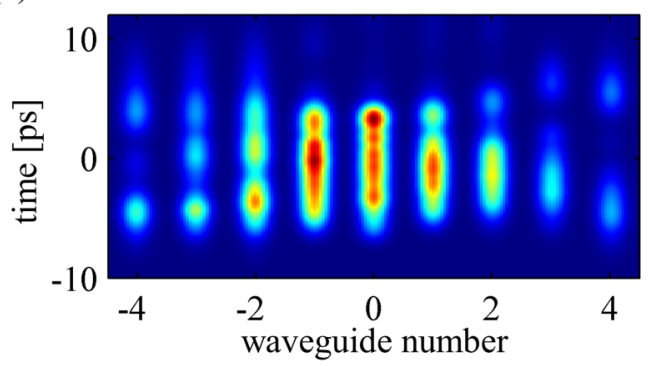

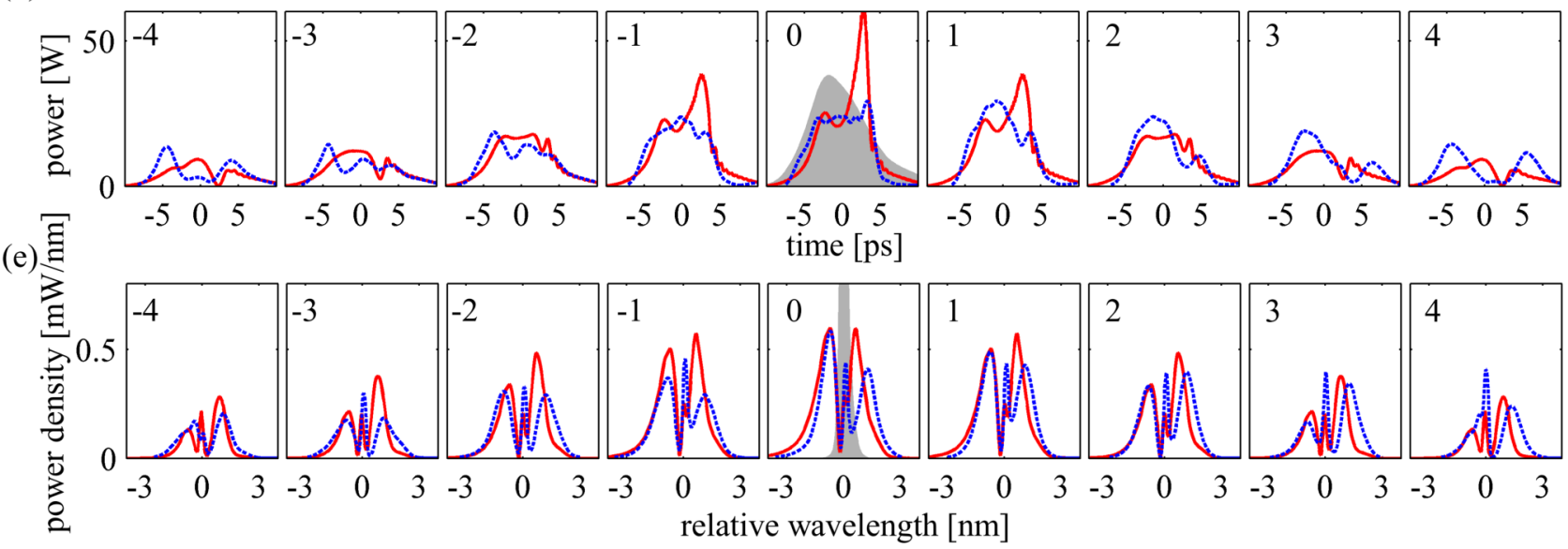

FIG. 4. (Color online) Results for effective focusing nonlinearity. (a) Power dependence of time-averaged spatial FW output of WGA. (b) Comparison of spatial outputs of measurement (blue solid line), corresponding to a cut trough data plotted in (a), and simulation (red circles) for input peak power of $360 \mathrm{~W}$. (c) Measured output spatiotemporal power distribution for FW pulse with $360 \mathrm{~W}$ input peak power. (d), (e) Comparison of the measured (blue dotted line) and simulated (red solid line) (d) temporal and (e) spectral powers in the different waveguides (waveguide number is indicated in each plot). The power of the input pulse is plotted in the center waveguide as a gray area for comparison.

first investigate the case of focusing nonlinearity. This is the case when the input wavelength $\lambda^{\mathrm{FW}}=1561.8 \mathrm{~nm}$ is above the phase-matching wavelength and the normalized phase mismatch is positive; $\Delta \beta L=51 \pi$. The results of measurements and simulations for this case are summarized in Fig. 4. The time-averaged FW spatial output power distribution of the WGA vs the input peak power is shown in Fig. 4(a). For low input powers the beam diffracts, forming a wide Gaussian pattern at the WGA output. With the increase of the input power, the output beam is narrowing due to the increasing cascading nonlinearity, eventually forming a discrete spatial solitary wave at powers around $300 \mathrm{~W}$ [7]. The output field is analyzed in detail for the input peak power of $360 \mathrm{~W}$, which is $20 \%$ larger than the power required for soliton generation. Such power is sufficient to observe the effects discussed above, including the asymmetric pulse reshaping and pulse breakup in the outer waveguides. For this power the measured spatial profile of the output beam is compared to the simulated spatial output profile in Fig. 4(b), showing good agreement between experimental and numerical results.

The spatiotemporal structure of the FW field is determined by measuring FROG traces of nine waveguides centered around the beam maximum. The time-dependent power retrieved numerically from the measured FROG traces is shown in Fig. 4(c). Here, the transverse spatial structure of the waveguide modes is approximated by Gaussian distributions. In general the measured pulse behavior is similar to the pulse breakup in the simulations discussed in view of Figs. 3(a)-3(c).
At higher momentary powers, close to the temporal center of the pulse, the fields are spatially more localized than in the wings. Here, the beam focuses down to a slightly narrower spatial distribution than the input beam, confirming that the peak power is larger than the soliton power. This leads to temporal splitting of the pulses in the waveguides away from the beam center and to pulse compression in the central waveguide. A similar phenomenon was first described theoretically two decades ago [44] and recently confirmed experimentally [18] in two-core couplers. We note that such pulse splitting was also used in WGAs with Kerr nonlinearity to excite X-shaped waves $[10,11,45]$. Similar effects were also observed in bulk media with quadratic nonlinearity [46,47]; however, our work represents the first direct observation in quadratic nonlinear WGAs.

The measured and simulated temporal power distributions are directly compared in Fig. 4(d), and we find qualitative agreement between experiment (blue) and theory (red). We plot the input pulse in the central waveguide as a gray shading for comparison. The pulse splitting in the outer waveguides is clearly visible in both theoretical and experimental traces. Additionally, the pulse in the central waveguide is narrowed and skewed towards the trailing edge, as a signature of the cascaded nonlinearity limitations discussed above in Fig. 3(d). The back-switching of the pulse in the edge waveguide is visible; however, it is not as pronounced as in the simulations. The similarity between the pulses in the waveguides to the right and to the left of the beam center proves the reliability 
of our measurement approach and confirms that only small transverse inhomogeneities are present in the WGA.

The measured (red) and simulated (blue) spectra of the pulses in different waveguides are shown in Fig. 4(e). The plotted experimental spectra are measured with an OSA and agree well with the spectra retrieved from the FROG (not shown). Again we plot the spectrum of the input pulse (gray shading) for comparison. The measured and simulated output spectra are split into several peaks as known from the well-understood process of self-phase modulation [48]. The resulting considerable spectral broadening with respect to the input spectrum is obvious.

Although the deviations between the simulation and experiment are relatively small, they still need to be addressed. The origin of this mismatch is likely related to a small crystal nonuniformity. This nonuniformity has been measured with tuning curves of diffractionless propagating beams in the array and was included into the theory by variable propagation constants $\beta^{m}(z)$. However, small-scale inhomogeneities and defects of the waveguides as well as fluctuations of the coupling between the waveguides in the array are not measurable and hence cannot be included in the simulations. A potentially more serious reason for uncertainties in the coupling was the remaining photorefraction in the sample. Despite the high operation temperature of $230{ }^{\circ} \mathrm{C}$, the relatively strong $\mathrm{SH}$ wave induces small photorefractive effects. Thus, it influences the phase matching and the coupling between waveguides, hindering a perfect theoretical description of the system. Additionally, the measured average intensities in the outer waveguides are not very high, leading to an operation of the FROG with long integration times, resulting in noisy FROG traces. This affects the FROG retrieval algorithm, and therefore fine details of the pulses may not be completely retrieved.

\section{B. Defocusing nonlinearity}

Measurements equivalent to those in the case of focusing nonlinearity are also conducted for defocusing nonlinearity at a wavelength of $1553 \mathrm{~nm}$, below the phase-matching wavelength with a normalized phase mismatch of $\Delta \beta L=-53 \pi$. To obtain spatial focusing, staggered states at the edge of the Brillouin zone with transverse wave numbers of $k_{\perp} d= \pm \pi$ are excited by tilting the excitation beam [7]. For excitation at the edge of the Brillouin zone, the action of the effective defocusing nonlinearity leads to spatial localization inside the Bragg-reflection gap, resulting in the formation of a spatial gap soliton. Due to the lower coupling efficiency with tilted excitation, the highest peak power coupled to the WGA is lower than in the case of normal excitation. The input-power dependence of the WGA output-power distribution is shown in Fig. 5(a). Again we find spatial narrowing of the beam with increasing input power. For the highest measured peak power a spatial gap soliton is formed whose spatial structure agrees well with the simulation results, as shown in Fig. 5(b). The (a)

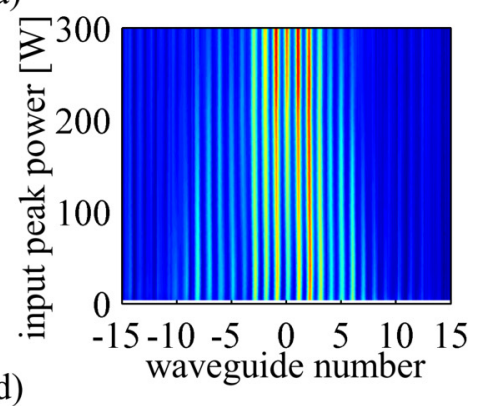

(b)

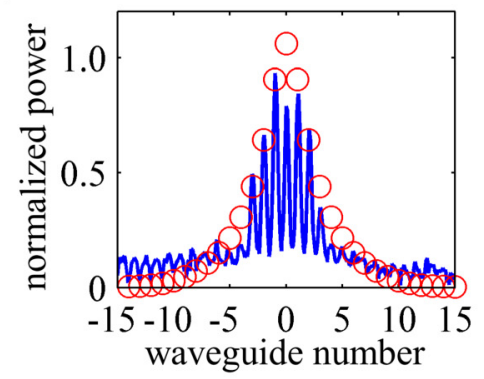

(c)

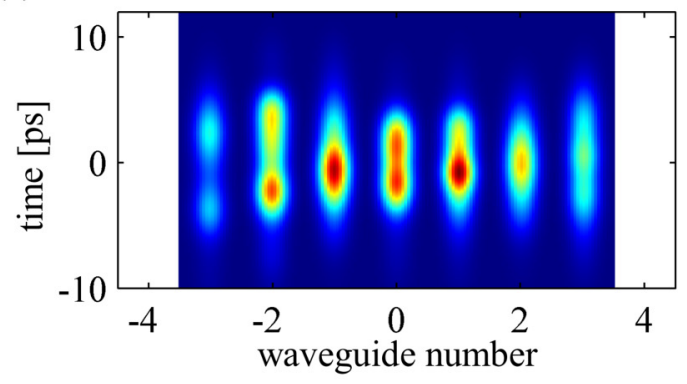

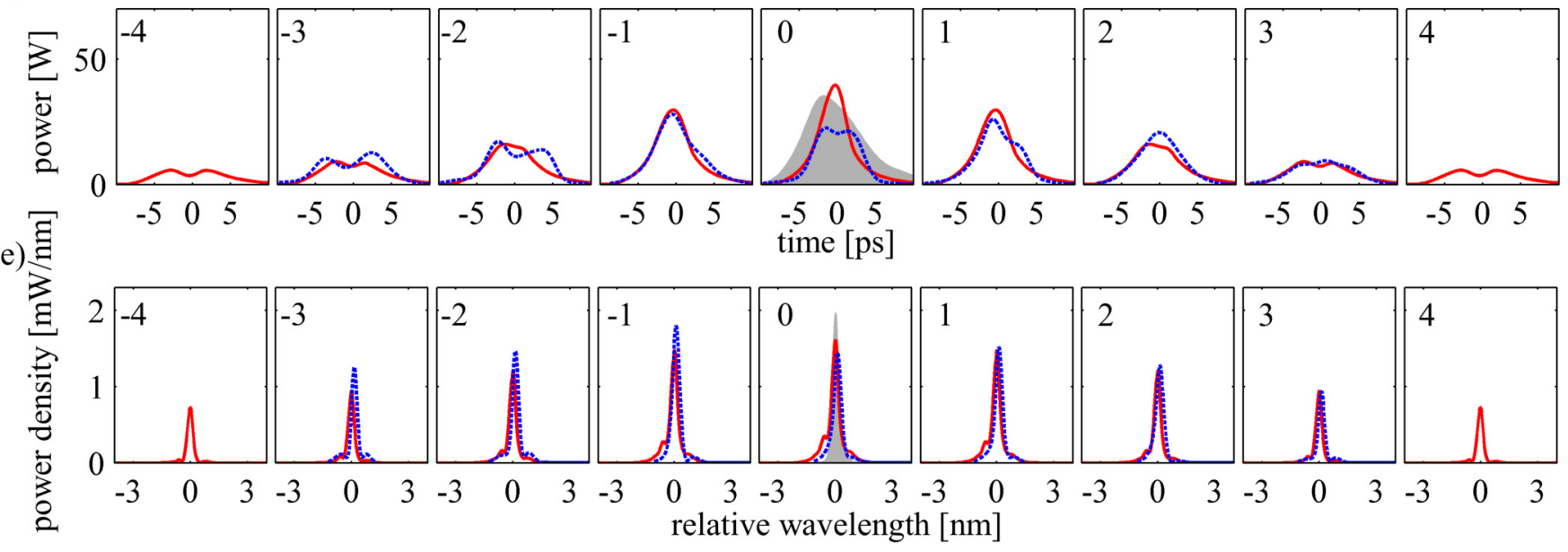

FIG. 5. (Color online) Result for effective defocusing nonlinearity. (a) Power dependence of spatial FW output of WGA. (b) Comparison of spatial outputs of measurement (blue solid line), corresponding to cut trough the data plotted in panel (a), and simulation (red circles) for input peak power of 300 W. (c) Measured output spatiotemporal power distribution for FW pulse with $300 \mathrm{~W}$ input peak power. (d), (e) Comparison of the measured (blue dotted line) and simulated (red solid line) (d) temporal and (e) spectral powers in the different waveguides (waveguide number is indicated in each plot). The power of the input pulse is plotted in the center waveguide as a gray area for comparison. 
maxima in the wings of the spatial distribution, which are localized between the waveguides, stem from higher-order linear FW bands excited by the tilted input beam. This is an artefact of the lower-excitation quality with the tilted input beam.

Figure 5(c) shows the measured spatiotemporal intensity distribution of the FW output field at an input power of $300 \mathrm{~W}$. Due to the lower input power, the FROG measurements only produced meaningful results for the seven central waveguides. Similar to the focusing case, we again observe pulse narrowing in the central waveguide. However, the splitting of the pulses is not as pronounced and can only be clearly observed in waveguides -2 and -3 . The same observation is made when comparing the measurement (blue) and the simulation results (red) for each waveguide in Fig. 5(d). Both experiment and simulation show a small dip between the two subpulses in the outer waveguides. In comparison with the focusing case, the measured output pulse shapes are much closer to the input pulse shape (gray shading).

Being very narrow and similar to the input spectra, the measured and simulated spectral distributions in Fig. 5(e) are distinctively different from the ones obtained in the focusing case. For increase of the input power the positive chirp of the input pulse is mitigated by the negative phase shifts of the self-phase modulation, leading to spectral narrowing. A peak power of $300 \mathrm{~W}$ is large enough to compensate the chirp from the input pulse, but does not overcompensate it, which again would yield spectral broadening [49,50].

Due to the missing spectral broadening, only negligible FW spectral components are shifted towards phase matching, and the strong temporal asymmetry of the output pulses [see Figs. 3 and 5(d)] is prevented. In this case the nonlinearity acts more as quasi-instantaneous. However, as we tested in simulations, for powers noticeably above the soliton power, spectral broadening occurs. Similar effects as for focusing nonlinearity are observed.

\section{SUMMARY}

In conclusion, we have investigated the spatiotemporal dynamics of ultrashort pulse propagation in WGAs with quadratic nonlinearity in the regimes of focusing and defocusing effective nonlinearity. We have revealed the physics of such effects as pulse breakup and solitary-wave decay for spatiotemporal pulse evolution. We have found that the cascaded nonlinearity is limited by the frequency dependence of the mode indices and by nonlinear induced phase matching, yielding FW losses due to SHG and degrading nonlinear refraction. These effects act as perturbations, limiting the formation of spatial solitary waves in quadratic nonlinear WGAs for powers above the soliton power. Similar effects are known for WGAs with an instantaneous Kerr nonlinearity where, upon propagation irregularities, spatial solitons will broaden, decay, or break up into several solitary entities [51].

\section{ACKNOWLEDGMENTS}

The authors gratefully acknowledge funding by the Australian Research Council, Discovery Projects (DP1093767), the German Academic Exchange Service (DAAD), the Group of Eight: Australia-Germany Joint Research Co-operation Scheme, the German Research Foundation DFG in SPP1391 Ultrafast Nanooptics, and the International Bureau of the German Federal Ministry of Education and Research.
[1] D. N. Christodoulides, F. Lederer, and Y. Silberberg, Nature (London) 424, 817 (2003).

[2] F. Lederer, G. I. Stegeman, D. N. Christodoulides, G. Assanto, M. Segev, and Y. Silberberg, Phys. Rep. 463, 1 (2008).

[3] J. Fleischer, G. Bartal, O. Cohen, T. Schwartz, O. Manela, B. Freedman, M. Segev, H. Buljan, and N. Efremidis, Opt. Express 13, 1780 (2005).

[4] C. R. Rosberg, D. N. Neshev, W. Krolikowski, A. Mitchell, R. A. Vicencio, M. I. Molina, and Y. S. Kivshar, Phys. Rev. Lett. 97, 083901 (2006).

[5] H. S. Eisenberg, Y. Silberberg, R. Morandotti, A. R. Boyd, and J. S. Aitchison, Phys. Rev. Lett. 81, 3383 (1998).

[6] D. Mandelik, H. S. Eisenberg, Y. Silberberg, R. Morandotti, and J. S. Aitchison, Phys. Rev. Lett. 90, 253902 (2003).

[7] R. Iwanow, R. Schiek, G. I. Stegeman, T. Pertsch, F. Lederer, Y. Min, and W. Sohler, Phys. Rev. Lett. 93, 113902 (2004).

[8] D. D. Hudson, K. Shish, T. R. Schibli, J. N. Kutz, D. N. Christodoulides, R. Morandotti, and S. T. Cundiff, Opt. Lett. 33, 1440 (2008).

[9] D. D. Hudson, J. N. Kutz, T. R. Schibli, Q. Chao, D. N. Christodoulides, R. Morandotti, and S. T. Cundiff, Phys. Rev. A 85, 031806 (2012).

[10] Y. Lahini, E. Frumker, Y. Silberberg, S. Droulias, K. Hizanidis, R. Morandotti, and D. N. Christodoulides, Phys. Rev. Lett. 98, 023901 (2007).
[11] M. Heinrich, A. Szameit, F. Dreisow, R. Keil, S. Minardi, T. Pertsch, S. Nolte, A. Tünnermann, and F. Lederer, Phys. Rev. Lett. 103, 113903 (2009).

[12] A. B. Aceves, G. G. Luther, C. De Angelis, A. M. Rubenchik, and S. K. Turitsyn, Phys. Rev. Lett. 75, 73 (1995).

[13] D. Cheskis, S. Bar-Ad, R. Morandotti, J. S. Aitchison, H. S. Eisenberg, Y. Silberberg, and D. Ross, Phys. Rev. Lett. 91, 223901 (2003).

[14] S. Minardi, F. Eilenberger, Y. V. Kartashov, A. Szameit, U. Röpke, J. Kobelke, K. Schuster, H. Bartelt, S. Nolte, L. Torner, F. Lederer, A. Tünnermann, and T. Pertsch, Phys. Rev. Lett. 105, 263901 (2010).

[15] F. Eilenberger, K. Prater, S. Minardi, R. Geiss, U. Röpke, J. Kobelke, K. Schuster, H. Bartelt, S. Nolte, A. Tünnermann, and T. Pertsch, Phys. Rev. X 3, 041031 (2013).

[16] S. M. Saltiel, A. A. Sukhorukov, and Y. S. Kivshar, Prog. Opt. 47, 1 (2005).

[17] F. Setzpfandt, A. A. Sukhorukov, D. N. Neshev, R. Schiek, A. S. Solntsev, R. Ricken, Y. Min, W. Sohler, Y. S. Kivshar, and T. Pertsch, Opt. Express 19, 23188 (2011).

[18] R. Schiek, A. S. Solntsev, and D. N. Neshev, Appl. Phys. Lett. 100, 111117 (2012).

[19] R. Iwanow, R. Schiek, G. Stegeman, T. Pertsch, F. Lederer, Y. Min, and W. Sohler, Opto-Electronics Rev. 13, 113 (2005). 
[20] F. Setzpfandt, M. Falkner, T. Pertsch, W. Sohler, and R. Schiek, Appl. Phys. Lett. 102, 081104 (2013).

[21] F. Setzpfandt, D. N. Neshev, R. Schiek, F. Lederer, A. Tünnermann, and T. Pertsch, Opt. Lett. 34, 3589 (2009).

[22] D. Mandelik, H. S. Eisenberg, Y. Silberberg, R. Morandotti, and J. S. Aitchison, Phys. Rev. Lett. 90, 053902 (2003).

[23] T. Pertsch, T. Zentgraf, U. Peschel, A. Bräuer, and F. Lederer, Phys. Rev. Lett. 88, 093901 (2002).

[24] J. A. Armstrong, N. Bloembergen, J. Ducuing, and P. S. Pershan, Phys. Rev. 127, 1918 (1962).

[25] M. Fejer, G. Magel, D. H. Jundt, and R. Byer, IEEE J. Quantum Electron. 28, 2631 (1992).

[26] R. DeSalvo, D. J. Hagan, M. Sheik-Bahae, G. Stegeman, E. W. Van Stryland, and H. Vanherzeele, Opt. Lett. 17, 28 (1992).

[27] M. L. Sundheimer, C. Bosshard, E. W. V. Stryland, G. I. Stegeman, and J. D. Bierlein, Opt. Lett. 18, 1397 (1993).

[28] R. Schiek, M. L. Sundheimer, D. Y. Kim, Y. Baek, G. I. Stegeman, H. Seibert, and W. Sohler, Opt. Lett. 19, 1949 (1994).

[29] R. Schiek, Y. Baek, and G. I. Stegeman, J. Opt. Soc. Am. B 15, 2255 (1998).

[30] R. Schiek, J. Opt. Soc. Am. B 10, 1848 (1993).

[31] T. Peschel, U. Peschel, and F. Lederer, Phys. Rev. E 57, 1127 (1998).

[32] O. Bang, P. L. Christiansen, and C. B. Clausen, Phys. Rev. E 56, 7257 (1997).

[33] M. Bache, O. Bang, J. Moses, and F. W. Wise, Opt. Lett. 32, 2490 (2007).

[34] M. Bache, J. Moses, and F. W. Wise, J. Opt. Soc. Am. B 24, 2752 (2007).

[35] D. Kane and R. Trebino, IEEE J. Quantum Electron. 29, 571 (1993).
[36] K. W. DeLong, R. Trebino, J. Hunter, and W. E. White, J. Opt. Soc. Am. B 11, 2206 (1994).

[37] K. W. DeLong, R. Trebino, and D. J. Kane, J. Opt. Soc. Am. B 11, 1595 (1994).

[38] R. Schiek and T. Pertsch, Opt. Mater. Express 2, 126 (2012).

[39] G. P. Bava, I. Montrosset, W. Sohler, and H. Suche, IEEE J. Quantum Electron. 23, 42 (1987).

[40] E. Strake, G. Bava, and I. Montrosset, J. Lightwave Technol. 6, 1126 (1988).

[41] G. J. Edwards and M. Lawrence, Opt. Quantum Electron. 16, 373 (1984).

[42] W. Press, S. Teukolsky, W. Vetterling, and B. Flannery, Numerical Recipes in C, 2nd ed. (Cambridge University Press, Cambridge, 1992).

[43] R. Schiek, AEÜ Int. J. Electron. Commun. 51, 77 (1997).

[44] R. Schiek, Opt. Quantum Electron. 26, 415 (1994).

[45] S. Droulias, K. Hizanidis, J. Meier, and D. Christodoulides, Opt. Express 13, 1827 (2005).

[46] O. Jedrkiewicz, J. Trull, G. Valiulis, A. Piskarskas, C. Conti, S. Trillo, and P. Di Trapani, Phys. Rev. E 68, 026610 (2003).

[47] G. Valiulis, V. Jukna, O. Jedrkiewicz, M. Clerici, E. Rubino, and P. DiTrapani, Phys. Rev. A 83, 043834 (2011).

[48] G. Agrawal, Nonlinear Fiber Optics, Optics and Photonics (Academic Press, New York, 2007).

[49] S. A. Planas, N. L. P. Mansur, C. H. B. Cruz, and H. L. Fragnito, Opt. Lett. 18, 699 (1993).

[50] M. Oberthaler and R. A. Hopfel, Appl. Phys. Lett. 63, 1017 (1993).

[51] Y. Silberberg, Opt. Lett. 15, 1005 (1990). 